\title{
Sete pontos para compreender o neomaterialismo
}

André Lemos'

http://orcid.org/0000-0001-9291-6494

\author{
Elias Bitencourt" \\ https://orcid.org/0000-0001-7366-6469 \\ I - Universidade Federal da Bahia. \\ Salvador (BA), Brasil. \\ II - Universidade do Estado da Bahia. \\ Salvador (BA), Brasil.
}

Resumo: Esse texto é uma resposta ao artigo "Neomaterialismo e Antropológicas" (Galáxia, n. 45) feito como uma crítica ao texto "Epistemologia da comunicação, neomaterialismo e cultura digital" (Galáxia, n. 43). O nosso entendimento principal é que parte das críticas ao neomaterialismo e ao conceito de antropocentrismo têm sua gênese no desconhecimento das premissas que orientam a virada material nas ciências sociais. Neste sentido, sem entrar em polêmicas desnecessárias sobre as divergências e deficiências dos artigos em debate, procuramos indicar de forma pedagógica sete pontos para compreensão da proposta neomaterialista nos estudos em comunicação.

Palavras-Chave: neomaterialismo, comunicação, epistemologia.

Abstract: Seven topics to understand new materialism - This text is a response to the article "Neomaterialismo e Antropológicas" (Galáxia, n. 45) written as a critic paper to the article "Epistemologia da comunicação, neomaterialismo e cultura digital" (Galáxia, n. 43). Our main understanding is that part of the criticisms of neomaterialism and the concept of anthropocentrism have their genesis in the ignorance of the premises that guide the material turn in the social sciences. In this sense, without entering into unnecessary polemics about the divergences and deficiencies of both articles under debate, we 
seek to indicate in a pedagogical way seven points for understanding the new materialism proposals in communication studies.

Keywords: new materialism, communication, epistemology

\section{Apresentação}

O objetivo desse texto é fornecer uma base introdutória e acessível aos principais conceitos e fundamentos que compõem as teorias neomaterialistas. Ele é uma resposta ao texto "Neomaterialismo e Antropológicas" (BRAGA, 2020), publicado nessa revista (Galáxia, n. 45) como uma crítica ao artigo "Epistemologia da comunicação, neomaterialismo e cultura digital" (LEMOS, 2020), na mesma revista (Galáxia, n. 43). Busca-se explicar conceitos e ideias que não tenham ficado muito claros e, com isso, minimizar eventuais ruídos teóricos que dificultam reconhecer o neomaterialismo e a crítica ao antropocentrismo enquanto posições epistemológicas pertinentes, complementares e úteis às investigações de fenômenos comunicacionais mais recentes. A estrutura do texto é direta, apresentando sete tópicos que tentam responder às questões mais frequentemente postas tanto no texto ao qual esse é uma réplica, quanto em debates sobre o tema do neomaterialismo.

Acreditamos que esse texto possa servir como um guia para os que se interessam pelo tema, elucidando questões recorrentes e oferecendo um guia bibliográfico. Não se pretende aqui convencer ou sustentar o imperativo dessa abordagem teórica. Toda teoria deve colocar suas ideias em confronto com outras e buscar, sem ser totalitária, explicar globalmente o que pretende investigar. Caso contrário, não seria uma teoria. Toda teoria deve ser submetida ao confronto de uma realidade situada. Criticar teorias ou sustentar suas qualidades não significa nem desqualificar posições, nem tentar impor (mesmo sendo isso impossível) uma maneira única de analisar os fenômenos. Nossa posição é, portanto, pedagógica, visando explicar aquilo que comumente é mal compreendido quando se critica o antropocentrismo, ou quando se quer valorizar a agência dos objetos não humanos. Sugerimos aos leitores a leitura dos dois textos que motivaram este debate.

\section{A virada material não é uma novidade nas ciências sociais}

Nas ciências humanas e na filosofia autores sustentam uma "virada material". Não estamos criando essa controvérsia no campo das ciências humanas. Estamos trazendo-a para ser pensada e levada a sério no campo da 
comunicação. Essa "virada material" é apontada por inúmeros autores desde o final dos anos 1980. Ela tem origem nos Estudos de Ciência e Tecnologia (STS), na Teoria Ator-Rede (TAR), na Ontologia Orientada a Objetos (OOO) ou Realismo Especulativo, no Realismo Agencial, nos estudos sobre a Materialidade da Comunicação, de origem alemã, no Pragmatismo e ficou genericamente conhecida pelo termo "neomaterialismo". As correntes neomaterialistas, portanto, não são homogêneas e não negam a importância da agência humana. Elas apontam para a necessidade de observar os fenômenos levando a sério as implicações dos humanos com as demais forças materiais (BARAD, 2007; BRAIDOTTI, 2013; BRYANT, 2011; DEVELLENES e DILLET, 2018; DELANDA, 1996; DOLPHIJN e TUIN, 2012; FOX; ALLDRED, 2017; GAMBLE, HANAN e NAIL, 2019; GARCIA, 2014; GUMBRECHT e PFEIFFER, 1994; HARMAN, 2011, 2012, 2016; JAMES, 1912; KITTLER, 1999; MEILLASSOUX, 2009; MILLER, 2005; VERBEEK, 2005; WHITEHEAD, 1978).

\section{A crítica ao antropocentrismo não implica objetocentrismo ou tecnocentrismo}

A vida social é produzida por coletivos compostos de humanos e não humanos. Não podemos entender o lugar do humano sem reconhecer a agência dos objetos, das coisas, dos artefatos, dos outros animais. A posição das visões neomaterialistas aplicadas aos estudos em comunicação, portanto, é do entendimento do lugar do humano nos processos sociocomunicacionais. A comunicação social, como se chama na área, seria assim "associal" - colocando ênfase na associação de coletivos híbridos - pois se dá como produto de mediações (radicais) que têm no humano um ponto de passagem obrigatório, mas não único. Sem reconhecermos as agências dos objetos, ou reduzi-las apenas aos direcionamentos dos sujeitos, não entenderemos esse mesmo sujeito no processo. Portanto, fazer coro à crítica ao antropocentrismo não implica investir em perspectivas objetocêntricas, tecnocêntricas ou tecnodeterministas. (COOLE; FROST, 2010; GRUSIN, 2015a; LATOUR, 2004, 2005, 2008, 2015).

\section{A crítica ao antropocentrismo não defende a exclusão do humano nos processos}

Quando se questiona sobre a agência dos objetos a partir da constatação de que eles foram feitos pelos humanos, e que por isso o neomaterialismo também reproduz o antropocentrismo, é preciso explicar que o que interessa 
para entender as dimensões associativas de qualquer coletivo é se perguntar sobre "o que um objeto faz depois que foi feito". O problema não está a montante apenas (na forma e nos princípios de construção dos objetos), mas também a jusante, nas consequências do que ele faz fazer em associação a outros objetos (incluindo aí os humanos). É nessa associação imanente e localizada agora com o objeto já feito, que podemos identificar o problema, a controvérsia e suas dimensões (LEMOS, 2020; LATOUR, 2004, 2005, 2008, 2015).

\section{Quatro dimensões básicas das abordagens neomaterialistas}

Embora as agendas sejam muitas, destacamos quatro pontos que sintonizam as visões neomaterialistas:

\subsection{Tudo tem uma vinculação material}

A investigação de toda e qualquer associação deve levar em conta as dimensões materiais que irão balizar discursos e ações. A agência não está apenas no polo do sujeito. Nessa perspectiva, o foco não deixa de ser a percepção sobre o efeito das coisas no sujeito, mas vincula-se, necessariamente, às condições materiais precisas e verificáveis a partir das quais os sujeitos e as coisas se afetam mutuamente em dada circunstância (BARAD, 2007; BENNET, 2010; BRAIDOTTI, 2013; GAMBLE \& NAIL, 2020; LATOUR, 2012; MARRES, 2012; PINK, ARDÈVOL \& LANZENI, 2016).

\subsection{Posição mais pragmática}

O fenômeno que se procura conhecer é discutido não pelo que ele é (essência), mas pelo modo como é instaurado. Isso implica abordar o fenômeno por uma espécie de engenharia reversa imanente, que tenta compreendê-lo a partir do que ele faz, de como faz e de quais condições de associação importam para que ele se instaure, evitando, assim, explicações transcendentes sustentadas em chaves amplas definidas a priori (BUCHER, 2018; FOX; ALLDRED, 2017; GRUSIN, 2015; JAMES, 1912, 1987; STENGERS \& LATOUR, 2015; LATOUR, 2005, 2012, 2015; THRIFT, 2005).

\subsection{Metodologias orientadas por análises planas/simétricas}

As análises se voltam para aquilo que se evidencia enquanto questão de interesse na produção do fenômeno. Evita-se eleger, a priori, chaves hermenêuticas que privilegiam uma epistemologia centrada naquilo que é exclusivo ao humano. Consequentemente, isso nada tem a ver com “hipervalorizar 
a técnica", tampouco "negar o humano na técnica". O desafio é como reconhecer os entrelaçamentos humano - não humano (coisas, objetos, outros animais) que produzem o fenômeno, as particularidades dessas relações e as condições favoráveis para que essas associações ocorram. Trata-se de investigar, de maneira situada, como os diferentes aspectos envolvidos na experiência observada produzem diferenças na construção do fenômeno investigado (BARAD, 2007; FOX; ALLDRED, 2018; JAMES, 1912, 1987; LATOUR, 2004, 2005, 2008, 2012; MOL, 2002; WHITEHEAD, 1978).

\subsection{Crítica ao antropocentrismo}

Esses três aspectos citados estruturam uma crítica ao que se convencionou chamar, nos estudos neomaterialistas, de antropocentrismo. Essa é uma crítica ao "modo de ver" o fenômeno orientado por perspectivas epistemológicas que, de maneira apriorística, tendem a privilegiar apenas leituras do fenômeno a partir de chaves voltadas à análise da experiência humana, minimizando outras formas de agência que não as emanadas do sujeito a partir de sua consciência, percepção ou propriocepção (affordances). Entendemos a comunicação (que interessa ao nosso campo, não aquela das partículas no interior dos átomos) como uma forma de associação que tem no humano um ponto de passagem obrigatório. Nesse sentido, estudos antropocêntricos inibem a visibilidade da complexidade da "complicação" humana nesses processos. Assim, contrário ao que se pensa, ao excluir os agenciamentos dos objetos não humanos, o antropocentrismo retira parte importante da inter-relação do humano nos coletivos (social), impedido de ver, justamente, como nós, humanos, estamos implicados nessas associações (COOLE; FROST, 2010; FOX; ALLDRED, 2017, 2018; GAMBLE, HANAN; NAIL, 2019; LEMOS, 2020).

\section{Olhar imanente para as dobras da técnica}

Latour diz que a vida social dos outros animais (e a nossa também) é (são) complexa(s), mas só a dos humanos é, além de complexa, complicada. Os não humanos que compõem o coletivo no qual os humanos estão envolvidos acrescentam complicações (que contêm dobras - pli em francês), que mantêm estabilizações. Complicar significa acrescentar camadas, promover desdobramentos que afetam o modo como produzimos a vida em sociedade. A posição neomaterialista explora essas dobras tecnológicas como estratégia metodológica para interrogar o humano que também se produz ao produzi-las. As redes sociotécnicas são geradoras dessas complicações, 
desde os primeiros artefatos até os complexos sistemas algorítmicos contemporâneos. Pela perspectiva neomaterialista, cabe investigar de forma imanente essas dobras em controvérsias particulares (LATOUR, 2015).

\section{Gramáticas novas impõem revisões epistemológicas}

As teorias neomaterialistas servem para qualquer fenômeno coletivo e não são específicas para análises da cultura digital. No entanto, o texto criticado de origem discutia mais a cultura digital. Assim sendo, podemos dizer que o contexto das plataformas digitais e o da dataficação da vida, por exemplo, expõem essas dobras dos novos objetos infocomunicacionais. Ao ser incorporada à infraestrutura material dos novos meios digitais, a ação de algoritmos passa a exercer alcance amplo e diversificado em diferentes esferas do social (informação, saúde, governança, entretenimento, mobilidade urbana, esferas de intimidade etc.). Esses eventos acrescentam camadas de complicação aos fenômenos comunicacionais na medida em que não só trazem variáveis novas (predições, acessos ao bem comum regulados por algoritmos, processos de invisibilidade promovidos por sistemas de recomendação das redes sociais), como ampliam a escala do protagonismo desses elementos técnicos que formam a infraestrutura do ecossistema midiático atual (BUCHER, 2018; MARRES, 2017; PINK, ARDÈVOL; LANZENI, 2016; MARRES; GERLITZ, 2015; MORTON, 2013).

\section{A crítica ao antropocentrismo na comunicação é propositiva, não antagonista}

A crítica ao antropocentrismo na comunicação não parte de um antagonismo epistemológico às correntes tradicionais, mas sobretudo da necessidade de pensarmos a respeito dos limites que elas trazem para entendermos as traduções do fenômeno comunicacional. Por serem orientadas à exposição das evidências situadas que compõem os fenômenos investigados, as perspectivas neomaterialistas podem contribuir para pensar criticamente os caminhos epistemológicos diante das questões atuais da comunicação. Nesse sentido, as aproximações neomaterialistas aos estudos de mídia ou à crítica ao antropocentrismo na comunicação não visam "desqualificar" outras perspectivas ou o histórico do campo no país, nem tampouco impor uma visão única a ser adotada (BENNETT, 2010; BRAIDOTTI, 2013; FOX \& ALLDRED, 2017; GRUSIN, 2015; HARMAN, 2011, 2012, 2016; LATOUR, 2005; LEMOS, 2020; PARIKKA, 2012; PEREIRA, BELEZA; AZEVEDO, 2019; VERBEEK, 2005). 


\section{Contribuição do debate à área da comunicação no Brasil}

Acreditamos que a abordagem neomaterialista, ainda pouco conhecida no Brasil, atestando um descompasso com outras áreas das ciências sociais, deve ser estudada e levada a sério. Descartes sumários não ajudam o enriquecimento epistemológico do campo da comunicação no país. Com esses sete pontos buscamos tornar mais compreensíveis as principais características das teorias neomaterialistas e incentivar o interesse pelo tema. Acreditamos que essa perspectiva teórico-metodológica pode ser de grande ajuda para diversos estudos no campo das ciências sociais, e não apenas aqueles referentes à cultura digital. As referências colocadas no final de cada tópico compõem um mapa sugerido de leitura importante para o aprofundamento das questões teóricas e metodológicas vinculadas ao neomaterialismo e abordagens não antropocêntricas.

Propostas teóricas devem ser criticadas, mas com seriedade, com análise de seus textos canônicos, de suas referências mais importantes e de suas análises empíricas. Certamente há problemas com as teorias neomaterialistas e estes poderiam ser apontados com relação a outras teorias da comunicação ou utilizadas pelo "campo da comunicação". O dever de casa deve ser feito. Cabe aos pesquisadores da área se dedicarem a esse trabalho, caso queiram criticá-las ou acolhê-las.

André Lemos é professor titular da Faculdade de Comunicação da UFBA e professor do Programa de PósGraduação em Comunicação e Cultura Contemporâneas da UFBA; coordena o Lab404.

almlemos@gmail.com

Elias Bitencourt é professor assistente na Universidade do Estado da Bahia e Professor do Programa de PósGraduação em Comunicação e Cultura Contemporâneas da UFBA.

eliasbitencourt@gmail.com

Contribuição de cada co-autor: ambos escrita, revisão e edição; fundamentação teórica e conceituação. 


\section{Referências}

BARAD, K. Meeting the Universe Halfway, p. 39-70, 2007. Disponível em: <https://read. dukeupress.edu/books/book/1068/chapter/151817/>.

BENNET, J. Vibrant Matter: A Political Ecology of Things. Durham, London: Duke University Press Durham, NC, 2010. Disponível em: <http://linkinghub.elsevier.com/retrieve/pii/ S175545861000054X>.

BRAIDOTTI, R. The Posthuman. Cambridge, UK: Polity Press, 2013.

BRAGA, J. L. Neomaterialismo \& antropológicas. Galáxia, n. 45, set-dez, 2020, p. 20-33.

BRYANT, L. The Democracy of Objects. Michigan. Open Humanities Press, 2011

BUCHER, T. If... Then Algorithimic Power and Politics. New York: Oxford University Press, 2018.

COOLE, D.; FROST, S. New Materialisms_ Ontology, Agency, and Politics. Durham \& London: Duke University Press, 2010.

DELANDA, M. The Geology of Morals. A Neo-Materialist Interpretation. 1996. Disponível em: <http://www.t0.or.at/delanda/geology.htm>.

DEVELLENES, C.; DILLET, B. Questioning New Materialisms: An Introduction. Theory, Culture \& Society, O(0) 1-16, 2018.

DOLPHIJN, R, TUIN, I. New materialism: interviews \& cartographies. Ann Arbor: Open Humanities Press, 2012.

FOX, N. J.; ALLDRED, P. Sociology and the New Materialism. Theory, Research, Action. London, England: SAGE Publications, 2017.

New materialism. In: Atkinson, P.A., Delamont, S., Hardy, M.A. and Williams, M. (eds.) The SAGE Encyclopedia of Research Methods. London: Sage, 2018.

GAMBLE, C. N.; HANAN, J. S.; NAIL, T. What Is New Materialism? Angelaki - Journal of the Theoretical Humanities, v. 24, n. 6, p. 111-134, 2019.

GAMBLE, C. N.; NAIL, T. Black Hole Materialism? Rhizomes: Cultural Studies in emerging Knowledge, no 36, 2020, doi:10.20415/rhiz/036.e08

GARCIA, T. Form and object: a treatise on things. Edinburgh: Edinburgh University Press, 2014.

GUMBRECHT, H. U; PFEIFFER, K. L. Materialities of Communication. Stanford: Stanford University Press, 1994.

GRUSIN, R. (ed.), The Nonhuman Turn. Minneapolis: University of Minnesota Press, 2015. . Radical Mediation. Critical Inquiry, v. 42, n. 1, p. 124-148, $2015 a$. 
HARMAN, G. The quadruple object. Hants, UK: Zero Books, 2011.

Weird realism: Lovecraft and philosophy. [S.I.]: John Hunt Publishing, 2012.

Immaterialism: Objects and Social Theory. Kindle ed. Cambridge: Polity Press, 2016.

JAMES, W. A pluralistic universe. William James Writings 1902-1910. New York: The Library of America, 1987.

Essays in Radical Empiricism. New York, London: Longmans, Green, and Co, 1912.

KITTLER, F. A. Gramophone, Film, Typewriter. Stanford: Stanford University Press, 1999.

LATOUR, Bruno. Why Has Critique Run out of Steam? From Matters of Fact to Matters of Concern. Critical Inquiry, v. 30, n. 2, p. 225-248, 2004. Disponível em: <http://www. journals.uchicago.edu/doi/10.1086/421123>.

Reassembling the social: An introduction to actor-network-theory. Oxford: Oxford university press, 2005.

What is the style of matters of concern? Spinoza Lectures. [S.I.]: Van Gorcum, 2008. p. $1-50$.

Enquête sur les modes d'existence: une anthropologie des Modernes. Paris: Éditions La Découverte, 2012.

Uma Sociologia Sem Objeto? Revista Valise, v. 5, n. 10, p. 165-187, 2015.

LEMOS, A. Epistemologia da comunicação, neomaterialismo e cultura digital. Galaxia (Online), v. 404, n. lii, p. 54-66, 2020. Disponível em: <https://revistas.pucsp.br/galaxia/ article/view/43970/31631>.

MARRES, N. Material Participation: Technology, Environment and Everyday Publics. Basingstoke: Palgrave Macmillan. 2012.

2017.

Digital Sociology: The reinvention of Social Research. Cambridge, UK: Polity Press,

MARRES, N.; GERLITZ, C. Interface Methods. Media Studies, n. 2011, p. 1-32, 2015.

MEILLASSOUX, Q. After Finitude. An Essay on the Necessity of Contingency. Continuum, London, 2009.

MILLER, D. Materiality. Durham, London: Duke University Press Durham, NC, 2005.

MOL, A. The Body Multiple: Ontology in Medical Practice. Durham \& London: Duke University Press, 2002.

MORTON, T. Hyperobjects: philosophy and ecology after the end of the world. Minneapolis: University of Minnesota Press., 2013. 
PARIKKA, J. New materialism as media theory: medianatures and dirty matter. In: Communication and Critical/Cultural Studies, Vol. 9, No. 1, March 2012, pp. 95-100

PEREIRA, C.; BELEZA, J.; AZEVEDO, M. As "coisas" em seus devidos lugares: as materialidades no campo da Comunicação e do Consumo. Revista E-compós, v. 23, jan-dez, p. 1-21, 2019.

PINK, S.; ARDÈVOL, E.; LANZENI, D. Digital materialities: Design and anthropology. [S.I.]: Bloomsbury Publishing, 2016.

STENGERS, I.; LATOUR, B. The Sphinx of work. The different modes of existence. [S.I: s.n.], 2015. p. 11-87.

THRIFT, N. Beyond mediation: three new material registers and their consequences. Materiality, p. 231-256, 2005.

VERBEEK, P. What Things Do. Philosophical Reflections on Technology, Agency, and Design. Pennsylvania: Pennsylvania State University Press, 2005. v.1.

WHITEHEAD, A. N. Process and Reality. Nova York: Free Press, 1978.

Ensaio recebido em 25/11/2020 e aprovado em 23/12/2020. 